\title{
Integrated photometric redshifts and SED fitting as tool for galaxy evolution studies
}

\author{
Ralf Kotulla \\ Center for Gravitation, Cosmology, and Astrophysics, Department of Physics, University of \\ Wisconsin-Milwaukee, 1900 E. Kenwood Blvd, Milwaukee, WI 53211, USA \\ email: kotulla@uwm.edu
}

\begin{abstract}
Photometric redshifts, i.e. redshifts derived by comparing an observed spectralenergy distribution (SED) to a range of empirical or theoretical SED templates, are commonly used in studies of the high-redshift Universe. Often, the next step is to use these redshifts as fixed input parameters for SED fitting to derive physical properties for each galaxy. However, this two-step approach ignores degeneracies between redshift and, e.g., stellar mass. Here I present first results using an improved approach that integrates both methods. I find that mass determinations are, on average, three times more uncertain than they seem from the common two-step approach. If not accounted for, these underestimated uncertainties can impact our ability of making meaningful comparisons between observations and simulations of galaxy evolution.
\end{abstract}

Keywords. methods: data analysis, techniques: photometric, galaxies: distances and redshifts, galaxies: high-redshift

\section{Introduction}

One key topic in current extragalactic research is the question as to how galaxies evolve over cosmic times, from forming the first stars in the early Universe to the manifold we observe in the local Universe. A popular approach to addressing this question is to study and compare galaxy populations at different redshifts and hence look-back times. Since the original Hubble Deep Field, many fields have been established with deep photometric observations across the full electromagnetic spectrum, extending our inventory of galaxies to fainter magnitudes, higher redshifts, and larger numbers, thus improving statistics while reducing measurement uncertainties. However, most galaxies are too faint for spectroscopic studies, so that we have to rely on their spectral-energy distributions (SEDs) alone to derive even basic quantities such as their redshifts. Fortunately, most popular fields have extensive multi-wavelength coverage, extending from the ultraviolet to the mid-infrared regime. These data are also the foundation to infer their physical properties, such as redshifts, stellar masses, star-formation rates (SFRs), and many others through the popular method of SED fitting.

Despite the inherent similarities of many of the photometric-redshift (photo- $z$ ) techniques (for an overview and comparison see, e.g., Hildebrandt et al. 2010) and SED-fitting approaches, these are usually applied independently. In addition, if stellar population models are used as templates, different basic assumptions, e.g., about the star-formation histories (SFHs), and/or physical ingredients, e.g., with respect to stellar evolution data, are made individually for each of the photo- $z$ and SED-fitting steps. This not only introduces inconsistencies, but also neglects degeneracies among, e.g., redshift, SFH, SFR, and dust extinction. Here we present first results from a novel approach aimed at integrating the 'traditional' SED fitting with a photo- $z$ code that fully and self-consistently 
accounts for all these degeneracies, yielding best-fitting parameters and uncertainties for the photometric redshifts as well as for a range of physical properties.

\section{Method, Data, and Results}

Our code GAZELLE is based on a $\chi^{2}$ minimization algorithm that compares the observed SED to a large number of theoretical templates. The template grid was computed using GALEV evolutionary synthesis models (Kotulla et al. 2009), which use a Salpeter stellar initial mass function and span a wide range of SFHs, from exponentially declining SFRs and SFRs that depend on the available gas mass (resembling a Schmidt-Kennicutt relation) to models including active and past starbursts. All models have been computed for a range of metallicities from $0.2 \mathrm{Z}_{\odot}$ to solar (for details about the importance of metallicity for photo- $z$ determinations, see Kotulla \& Fritze 2009) and include emission lines with line ratios appropriate for each metallicity. Assuming a formation redshift of $z_{\text {form }}=8$, we redshift each model according to its age and apply corrections for intergalactic HI attenuation (Madau 1995). Free parameters in the SED/photo- $z$ fitting routines are (i) the redshift, (ii) a normalization factor governing the stellar mass and all other mass-dependent parameters, and (iii) the intrinsic dust reddening (Calzetti et al. 2000).

As a test case we use data from the COSMOS field, covering the far-ultraviolet $(\sim 1500 \AA)$ to mid-infrared $(8 \mu \mathrm{m})$ spectral range with a total of 28 filters, including 12 optical intermediate-band filters. For validation we compare our photo- $z$ results with a sample of spectroscopic redshifts from the zCOSMOS survey (Lilly et al. 2007). We find a scatter of $\sigma_{z}=\left(z_{\text {spec }}-z_{\text {phot }}\right) /\left(1+z_{\text {spec }}\right) \approx 0.015$ and an outlier fraction (defined as the fraction of objects with photo- $z$ deviating from the corresponding spectroscopic redshift by $>3 \sigma)$ of $\sim 5 \%$, comparable to results from other groups (e.g., Ilbert et al. 2009).

Fig. 1 shows the full probability density functions (PDFs) for a redshift $z \sim 1$ galaxy from the COSMOS field. The probability density at each redshift point is computed from the minimum $\chi^{2}$ at this point using $P(z)=\exp \left(-\chi^{2} / 2\right) / P_{0}$, where $P_{0}$ is the normalization factor, to ensure that $\int P(z) \mathrm{d} z=1$. Uncertainties are derived from the minimum and maximum values encountered while integrating the PDF, sorted from highest to lowest, until we reach $68 \%$ confidence limits. Grey-shaded regions in Fig. 1 show the $1 \sigma$ ranges for each parameter.

The top panel of Fig. 1 shows the PDF as function of photo- $z$. It is obvious that different SFHs, shown by the differently colored lines, yield different best-fitting redshifts. In this case, the best-fitting photo- $z$ is very close to the spectroscopic redshift $\left(z_{\text {phot }}=\right.$ 0.9817 versus $\left.z_{\text {spec }}=1.0048\right)$, with $1 \sigma$ limits of 0.700 and 1.211 . The middle panel shows the corresponding PDF as a function of stellar mass. Clear differences from the top panel are that different SFHs correspond to different stellar masses, although (for the starburst/postburst models) the $\operatorname{PDF}(z)$ is quite similar. In addition, the $\operatorname{PDF}$ (mass) of each model is clearly more asymmetric than its $\operatorname{PDF}(z)$, since the distance modulus at these redshifts does not scale linearly with redshift. The bottom panel of Fig. 1 shows the PDF as a function of the instantaneous SFR. Here the different starburst models clearly separate, causing large uncertainties in the determination of the instantaneous SFR.

Taken together, the three panels of Fig. 1 clearly illustrate that uncertainties in the determination of the photometric redshift affect the precision of all other parameters. This error propagation leads to generally larger uncertainties that have to be properly accounted for when comparing observations to, for example, semi-empirical models of galaxy evolution (e.g., De Lucia \& Blaizot 2007). 

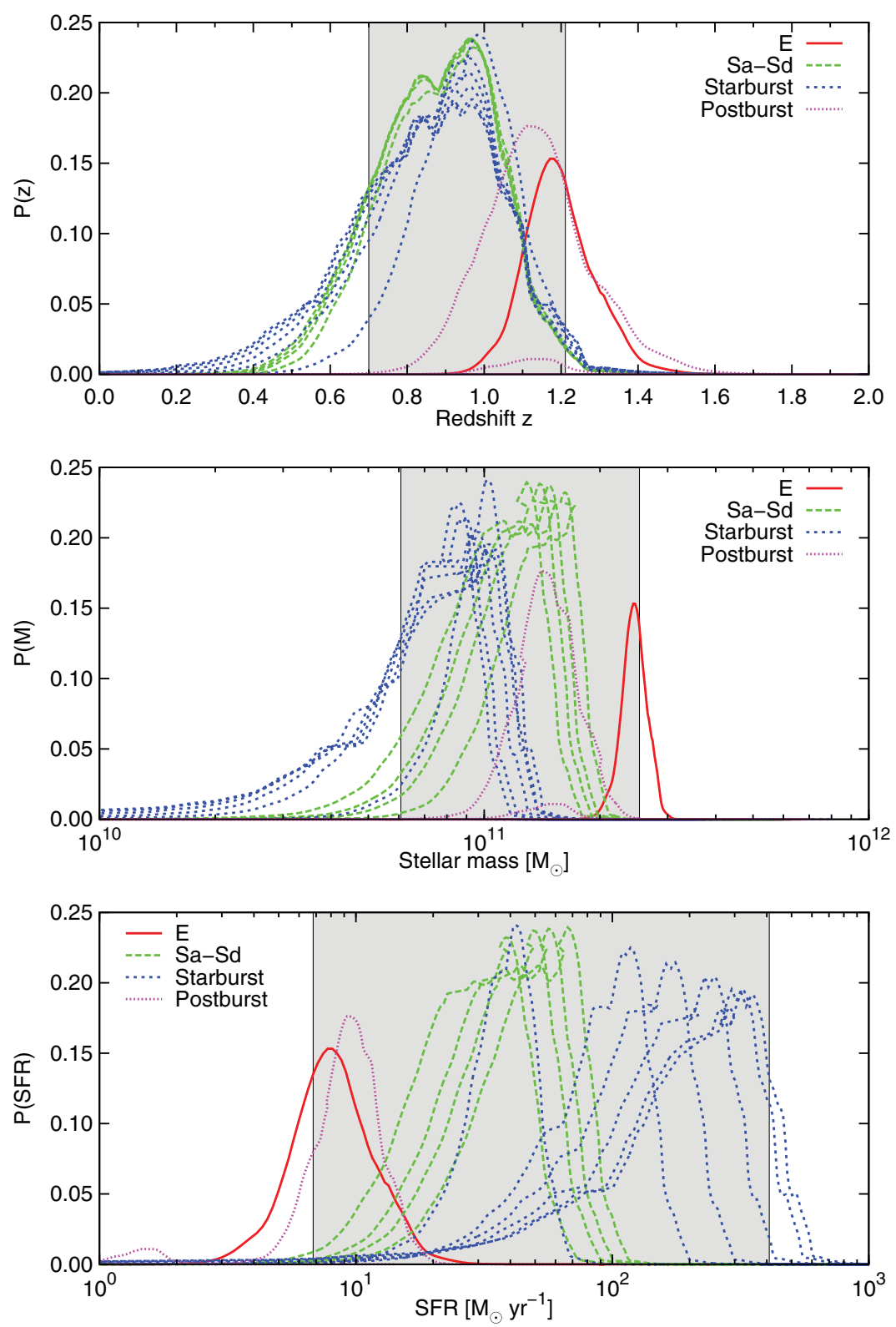

Figure 1. Probability density functions for one galaxy in the COSMOS field (zCOSMOS ID: 000847486; R.A.: $9^{\mathrm{h}} 59^{\mathrm{m}} 7.44^{\mathrm{s}}$, dec: $2^{\circ} 35^{\prime} 50.48^{\prime \prime}$, spectroscopic redshift $\left.z_{\text {spec }}=1.0048\right)$ for three different physical parameters. (top) Photometric redshift; (middle) stellar mass; (bottom) SFR. The grey-shaded regions illustrate the computed $1 \sigma$ ranges for each parameter. The different lines represent different SFHs (E: exponentially declining; Sa-Sd: SFR proportional to available gas mass; for details, see Kotulla et al. 2009).

To achieve a more quantitative analysis of the impact of redshift uncertainties on the determination of stellar masses, in Fig. 2 we show the relative uncertainties $\delta M / M$ for the full sample of galaxies in the COSMOS survey with reliable spectroscopic redshifts from zCOSMOS. For the red curve we simulated the common practice of computing photo- $z$ 




Figure 2. Relative uncertainties, $\delta M / M$, in the mass determination of galaxies with spectroscopic redshifts in the COSMOS field. Red (open) histogram: Uncertainties derived from SED fitting at fixed photometric redshifts; blue (shaded) histogram: Uncertainties accounting for degeneracies with redshift.

first, followed by fitting SEDs with the derived photo- $z$ as fixed input parameters, while the blue histogram shows the uncertainties if we fully account for redshift uncertainties. For fixed redshifts, typical uncertainties are 0.1 dex or $25 \%$, mostly driven by uncertainties in the determination of the dust reddening and degeneracies with the SFH. However, if photo- $z$ errors are accounted for, this increases to $\sim 0.3$ dex or a factor of 2 , mostly owing to less well-constrained distance moduli. This also explains the asymmetry in the distribution and the relatively prominent tail to very uncertain stellar masses: at very low redshifts, even small changes in redshift correspond to large changes in distance modulus, directly affecting the best-fitting stellar masses.

\section{Summary}

Photometric redshifts and SED fitting are both mature tools commonly used in current extragalactic research. However, applying them independently instead of in an integrated approach may not only introduce inconsistencies, but - more importantly-ignores uncertainties in the redshift determination as a major source of uncertainty in all derived physical properties. Here we present a possible solution and find that, on average, masses and all mass-dependent parameters (e.g., SFRs) are uncertain by typically 0.3 dex, with uncertainties increasing toward lower redshifts.

\section{References}

Calzetti, D., Armus, L., Bohlin, R. C., Kinney, A. L., Koornneef, J., \& Storchi-Bergmann, T. 2000, ApJ, 533, 682

De Lucia, G. \& Blaizot, J. 2007, MNRAS, 375, 2

Hildebrandt, H., Arnouts, S., Capak, P., et al. 2010, A 6 A, 523, A31

Ilbert, O., Capak, P., Salvato, M., et al. 2009, ApJ, 690, 1236

Kotulla, R. \& Fritze, U. 2009, MNRAS, 393, L55

Kotulla, R., Fritze, U., Weilbacher, P., \& Anders, P. 2009, MNRAS, 396, 462

Lilly, S., Le Fèvre, O., Renzini, A., et al. 2007, ApJS, 172, 70

Madau, P. 1995, ApJ, 441, 18 